\title{
Citius, Altius, Fortius
}

As done routinely every year, Thomson Reuters reported the impact factors (IF) of the journals in SCl-E, in last June. According to this recent report, the IF of Anatolian Journal of Cardiology (AJC) is $\mathbf{0 . 9 2 7}$. There could not be any other prideful coincidence in the first time that I have the opportunity to address to you.

Without any comparison, it would be difficult to understand why this figure is so "prideful". Our previous IF was 0.755 . It can be easily calculated that the recent figure is $23 \%$ higher than the previous one. The graphic below depicts the annual changes in our IF. It can clearly be seen that we have a steeper inclination compared to last year.

What makes the above-mentioned success even more important is its effect on the ranking of AJC among Turkish scientific journals. With this new IF, we moved one step upwards to the $10^{\text {th }}$ place. Although among all medical journals, our place

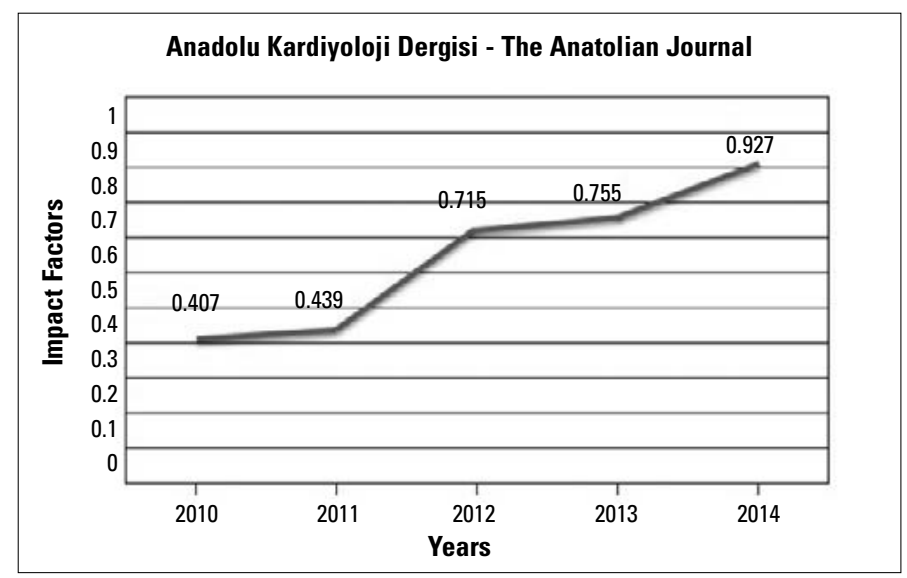

has not changed; we are at the $2^{\text {nd }}$ place in the ranking of journals of clinical sciences.

The euphoria of success must not prevent us from evaluating our current position with a critical eye. The readers of the journal are very familiar with the terms "relative" and "absolute" changes. In terms of absolute increment, our achievement is only a tiny 0.172 . We are still below the psychological threshold of " 1 ". To analyze this issue, it is best to go to web of science and overview AJC's impact on national and international cardiology. In the first half of the year 2015, our citations exceeded 2014 by $34 \%$. This is encouraging. However, almost $65 \%$ of these citations are from Turkish cardiologists. In other words, there is still lot to achieve to make AJC a more prominent journal globally.

You may wonder why I chose the Olympic motto as the title. It is simple. We have to be faster to move our journal to a higher position not among national but international journals. The only way to achieve this is to have a stronger scientific content.

To conclude, as the last one on board, I do not hesitate to give all the credit of this success to our chief editor and all the members of the editorial board. On behalf of the Turkish cardiology community, it is my duty to thank to all of them.

On the other hand, I can assure you that the editorial board of AJC, from top to bottom is very well aware of the new task to increase our impact on international cardiology.

Please keep in mind that the key to progress is to set a new task after every success.

\section{Zeki Öngen \\ Editor \\ İstanbul-Turkey}

